\title{
Ultrafast Spin Dynamics in Photodoped Spin-Orbit Mott Insulator $\mathrm{Sr}_{2} \mathrm{IrO}_{4}$
}

\author{
D. Afanasiev, ${ }^{1,}{ }^{*}$ A. Gatilova, ${ }^{2}$ D. J. Groenendijk, ${ }^{1}$ B. A. Ivanov, ${ }^{3}$ M. Gibert, ${ }^{4}$ S. Gariglio, ${ }^{4}$ J. Mentink, ${ }^{2}$ \\ J. Li ${ }^{5}$ N. Dasari, ${ }^{5}$ M. Eckstein, ${ }^{5}$ Th. Rasing, ${ }^{2}$ A. D. Caviglia, ${ }^{1}$ and A. V. Kimel ${ }^{2,6}$ \\ ${ }^{1}$ Kavli Institute of Nanoscience, Delft University of Technology, \\ P.O. Box 5046, 2600 GA Delft, Netherlands \\ ${ }^{2}$ Radboud University Nijmegen, Institute for Molecules and Materials, 6525 AJ Nijmegen, Netherlands \\ ${ }^{3}$ Institute of Magnetism, National Academy of Sciences and Ministry of Education and Science, \\ 03142 Kiev, Ukraine; \\ Faculty of Radio Physics, Electronics and Computer Systems, Taras Shevchenko National University of Kiev, \\ Kiev 01601, Ukraine; \\ and National University of Science and Technology "MISiS," Moscow, 119049, Russian Federation \\ ${ }^{4}$ DQMP, Université de Genève, 24 Quai E.-Ansermet 1211 Geneva, Switzerland \\ ${ }^{5}$ Department of Physics, University of Erlangen-Nuremberg, 91052 Erlangen, Germany \\ ${ }^{6}$ Russian Technological University, MIREA, Vernadsky Avenue 78, 119454, Moscow, Russian Federation
}

(Received 3 January 2018; revised manuscript received 27 February 2018; published 30 April 2019)

Ultrafast photodoping of the Mott insulators, possessing strong correlation between electronic and magnetic degrees of freedom, holds promise for launching an ultrafast dynamics of spins which cannot be described in terms of conventional models of ultrafast magnetism. Here we study the ultrafast laser-induced dynamics of the magnetic order in a novel spin-orbit Mott insulator $\mathrm{Sr}_{2} \mathrm{IrO}_{4}$ featuring an uncompensated pattern of antiferromagnetic spin ordering. Using the transient magneto-optical Kerr effect sensitive to the net magnetization, we reveal that photodoping by femtosecond laser pulses with photon energy above the Mott gap launches melting of the antiferromagnetic order seen as ultrafast demagnetization with a characteristic time of 300 fs followed by a sub-10-ps recovery. Nonequilibrium dynamical mean-field theory calculations based on the single-band Hubbard model confirm that ultrafast demagnetization is primarily governed by the laser-induced generation of electron-hole pairs, although the precise simulated time dependencies are rather different from the experimentally observed ones. To describe the experimental results, here we suggest a phenomenological model which is based on Onsager's formalism and accounts for the photogenerated electron-hole pairs using the concepts of holons and doublons.

DOI: 10.1103/PhysRevX.9.021020

\section{INTRODUCTION}

The manipulation of magnetic order at the fastest possible timescale is the ultimate goal of the rapidly developing area of ultrafast magnetism [1-4]. The purely fundamental interest in ultrafast magnetic phenomena is strengthened by its technological relevance and potential to impact technologies for recording and processing magnetically stored information.

The field of ultrafast magnetism started with the seminal discovery of subpicosecond demagnetization in metallic $\mathrm{Ni}$

\footnotetext{
*d.afanasiev@tudelft.nl
}

Published by the American Physical Society under the terms of the Creative Commons Attribution 4.0 International license. Further distribution of this work must maintain attribution to the author(s) and the published article's title, journal citation, and DOI.
Subject Areas: Condensed Matter Physics, Magnetism, Strongly Correlated Materials films after excitation with a sub-100-fs laser pulse [5]. Such a demagnetization was much faster than any interaction involving spins known at that time. Naturally, the discovery launched an intense search for relevant physical mechanisms and models which can explain the phenomenon. Despite numerous attempts, the majority of experiments on ultrafast demagnetization in metals performed in the past 20 years are still explained with the help of a phenomenological three-temperature (3T) model, in which the metal is seen as a system of three interconnected baths representing lattice, spins, and electrons, respectively [3]. According to this model, free electrons absorb laser radiation on a timescale faster than the spin-phonon interaction. This results in a sudden increase of their kinetic energy and thus an increase of the temperature to a very high value. The electron-phonon interaction leads to a consecutive increase of the temperature of the spin bath, seen as a melting of the magnetic order. Finally, interaction between hot electrons and the lattice results in their partial cooldown, and therefore a partial 
recovery of the magnetization. Obviously, in insulators, where electrons are not free and their kinetic energy cannot be large, such a mechanism of ultrafast laser-induced demagnetization is inefficient [6].

Mott insulators, however, may open a different chapter in ultrafast magnetism by revealing a new mechanism of picosecond demagnetization beyond the conventional $3 T$ model. These insulators are a special class of materials that should be metallic under conventional band theories, but are insulating due to strong on-site electron-electron repulsion. The minimal Hamiltonian which describes the physics of a Mott insulator is the Hubbard model, which includes a hopping term $t$ and an on-site Coulomb repulsion $U$ [7]:

$$
H=-t \sum_{\langle i j\rangle, \sigma} c_{i \sigma}^{\dagger} c_{j \sigma}+U \sum_{i} n_{i \uparrow} n_{i \downarrow}
$$

Here $c_{i \sigma}^{\dagger}$ and $c_{i \sigma}$ are creation and annihilation operators of electrons at site $i$ with spin $\sigma$ and $n_{i \sigma}=c_{i \sigma}^{\dagger} c_{i \sigma}$ is the electron occupancy at the site $i$. The summation $\langle i j\rangle$ extends over nearest neighbors. At half filling and in the limit $t \ll U$, the charge becomes localized to avoid the strong on-site repulsion, and the system is characterized by a single electron per site. The presence of localized electrons simultaneously with the insulating state implies the appearance of localized spins which have to be ordered at low temperature. Indeed, when electron repulsion is strong, the spins on adjacent sites are aligned oppositely [8]. The antiparallel spin alignment emerges similarly to the singlet state in the Heitler-London treatment if one considers the adjacent sites as those forming a covalent bond. Thus, at low temperatures in the Mott insulator the lattice sites hosting oppositely oriented spins form two interpenetrated antiferromagnetically coupled magnetic sublattices characterized by magnetizations $\mathbf{M}_{1}$ and $\mathbf{M}_{2}$, such that $\mathbf{M}_{1}=-\mathbf{M}_{2}$; see Fig. 1 .

Optical excitation of a Mott insulator with photon energies close to the Mott gap, i.e., photodoping, induces intersite charge transfer resulting in a hole on a lattice site (a holon) and a neighboring site with two electrons (a doublon); see Fig. 1(a). These hoppings are accompanied by intermixing of electron spins belonging to the oppositely oriented magnetic sublattices [Fig. 1(b)] and result in the formation of sites with zero magnetic moment $(S=0)$, seen as melting of the antiferromagnetic order. The subsequent motion of the photoinduced doublons and holons on the lattice implies a further reduction of the magnetic correlations, which can be interpreted as a transfer of kinetic energy from the photoinduced charges to the magnetic sector. The time of the electron delocalization is defined by the hopping parameter. As this time is in the range of $10 \mathrm{fs}$, this electron-hole-driven mechanism has been predicted to be relevant for laser-driven dynamics of both long-range magnetic order [9-11] and short-range spin correlations [12,13] in Mott insulators on the
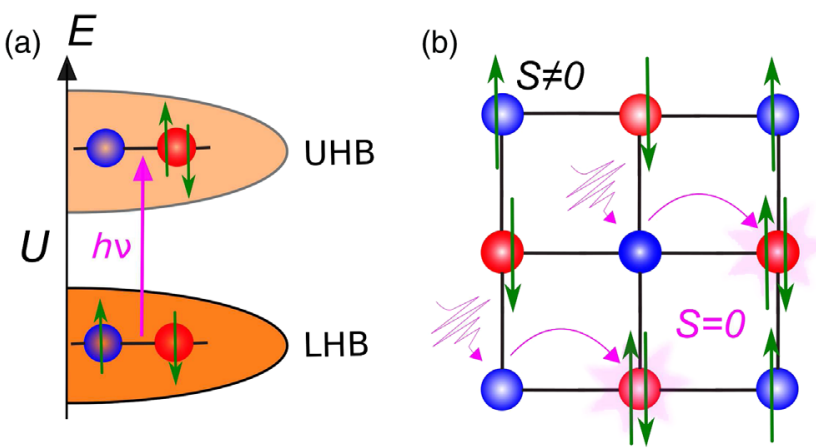

FIG. 1. (a) The band structure of a Mott insulator with schematics of the electronic configuration corresponding to the lower (LHB) and upper Hubbard bands (UHB). The pink arrow indicates an above the band gap optical excitation with photon having energy $h \nu$. (b) Magnetic structure of a Mott insulator subjected to the above band gap excitation. The staggered pattern of the blue and red circles denotes lattice sites hosting electrons having spins $S$ pointing "up" or "down," thus forming two oppositely orienting magnetic sublattices characterized by magnetizations $\mathbf{M}_{1}$ and $\mathbf{M}_{2}$, respectively. Pink arrows denote lightinduced hops of electrons between individual magnetic sublattices resulting in melting of the magnetic order.

picosecond of even subpicosecond timescales. Indeed, while only a nanosecond laser-induced dynamics has been reported for charge-transfer insulators [6,14], recent resonant inelastic x-ray scattering experiments on a Mott insulator reveal that ultrafast photodoping leads to a subpicosecond collapse of the macroscopic magnetic order [15].

Experimentally, the access to the magnetic order parameter in Mott insulators is hampered by the lack of a net magnetic moment in the collinear antiferromagnetic ground state. Experimental studies of ultrafast magnetism in Mott insulators are scarce, as to probe the antiferromagnetic order they require demanding experimental facilities with limited time resolution, such as free-electron lasers $[15,16]$. In so-called weak ferromagnets, strong spin-orbit coupling in combination with a lack of inversion symmetry cants the otherwise collinear antiferromagnetic magnetizations, such that although $\left|\mathbf{M}_{1}\right|=\left|\mathbf{M}_{2}\right|$, their cross product acquires a nonzero value such as $\mathbf{M}_{1} \times \mathbf{M}_{2} \neq 0$. This results in the emergence of a small net magnetization $\mathbf{M}=\mathbf{M}_{1}+\mathbf{M}_{2} \neq 0$. The magnitude of this magnetization can be directly probed with tabletop techniques using a probe sensitive to the macroscopic magnetization, such as the linear magneto-optical Kerr effect (MOKE) [17] or superconducting quantum interference device (SQUID) magnetometry. Since the net magnetic moment is coupled to the antiferromagnetic order, such measurements serve as an effective way to probe magnetic order in antiferromagnets with strong spin-orbit coupling.

Iridates represent a novel class of $5 d$ Mott insulators where the very opening of the insulating gap is mediated by the strong spin-orbit coupling. In $\mathrm{Sr}_{2} \mathrm{IrO}_{4}$, quasi-twodimensional member of this family, the elementary 
magnetic moments, contrary to conventional Mott insulators, but similarly to weak ferromagnets, are canted on large angle such that a net magnetic moment appear [18]. Therefore, this novel system enables prominent opportunities for tabletop magneto-optical studies of the entangled spin and charge dynamics of correlated oxides driven by ultrafast photodoping. Although magnetospectroscopic techniques were applied already to study the spectrum of magnetic excitations $[19,20]$, no time-resolved transient linear magneto-optics in $\mathrm{Sr}_{2} \mathrm{IrO}_{4}$ was demonstrated until now.

In this paper, we exploit the net magnetization of $\mathrm{Sr}_{2} \mathrm{IrO}_{4}$ to explore ultrafast magnetism in a Mott insulator. The photodoping of $\mathrm{Sr}_{2} \mathrm{IrO}_{4}$ by femtosecond laser pulses with photon energy above the Mott gap drives the dynamics of spins and charges. The magnetization dynamics probed by the MOKE appears to be substantially different from the charge dynamics probed by the photoinduced changes in intensity of the light reflected from the sample. Supported by the theory consideration, we argue that coupling between the net magnetization and the underlying antiferromagnetic order is present even during the out of the equilibrium photoinduced dynamics. Following this assumption, we demonstrate that ultrafast demagnetization seen in the MOKE reveals a melting of the antiferromagnetic order. The melting occurs on a characteristic time of about $300 \mathrm{fs}$ and is followed by a sub-10-ps recovery. While a temperature increase hardly affects the reflectivity dynamics, it results in a significant slowing-down of the dynamics of the magneto-optical signal.

To analyze the mechanism for the ultrafast melting in a spin-orbit Mott insulator, we perform nonequilibrium dynamical mean-field theory (DMFT) simulations of the laser-induced dynamics for the case of a minimalistic oneband Hubbard model with the parameters deduced from the electronic structure of $\mathrm{Sr}_{2} \mathrm{IrO}_{4}$. While this model is clearly too simplistic to account for all experimental time dependencies, the simulations clearly reveal a subpicosecond melting of the magnetic order. They confirm that the mechanism of electron-hole-driven melting of antiferromagnetism, which has been predicted in simple Mott insulators $[12,13]$, can also account for the early stage of the demagnetizations in a spin-orbit coupled Mott insulator. The remaining discrepancies call for more complex DMFT calculations that are technically very challenging if not impossible. To describe the experimental data, therefore, we also propose a novel phenomenological approach which is based on Onsager's formalism and accounts for the role of the photogenerated electron-hole pairs using the concepts of holons and doublons. In the model, the ultrafast demagnetization is primarily governed by the impact photoionization process. It results in an excess amount of the holons and doublons leading to ultrafast loss of magnetic order. The recovery dynamics is a result of the relaxation of the magnetic system into the state corresponding to thermodynamic equilibrium and thus predominantly defined by the dependence of the free energy on magnetization.

The paper is organized as follows. In Sec. II, we consider a spin-orbital Hamiltonian, which has recently been suggested to describe $\mathrm{Sr}_{2} \mathrm{IrO}_{4}$ and analytically show that within this Hamiltonian the canted magnetic order as it is present in $\mathrm{Sr}_{2} \mathrm{IrO}_{4}$ can be effectively mapped onto the collinear order inherent to a regular Mott state. Here we also theoretically argue why the ferromagnetic and antiferromagnetic order parameters remain coupled during outof-equilibrium photoinduced dynamics. In Secs. III and IV, we present extensive structural, magnetic, and magnetooptical characterizations of $\mathrm{Sr}_{2} \mathrm{IrO}_{4}$ thin films. In Sec. V, we investigate the ultrafast dynamics of the MOKE and reflectivity triggered by an ultrashort pulse of visible light and compare experimental results with the nonequilibrium DMFT simulations. In Sec. VI, we present the results of the modeling of the ultrafast dynamics using Onsager's formalism. Finally, we summarize the results in Sec. VII.

\section{MAPPING OF THE $\boldsymbol{J}_{\text {eff }}=1 / 2$ HUBBARD MODEL TO THE REGULAR HUBBARD MODEL}

The recent theoretical and experimental results confirm that magnetism of $\mathrm{Sr}_{2} \mathrm{IrO}_{4}$ is determined by the effective total angular momentum $J_{\text {eff }}=1 / 2$ rather than solely by the spin magnetic moment $S=1 / 2$ [21,22]. The elementary magnetic moments of iridium ions are aligned in a staggered pattern such that it is possible to allocate two interpenetrating magnetic sublattices denoted " 1 " and " 2 ". The strong spin-orbit coupling mutually cants the moments situated on neighboring lattice sites on an angle $2 \theta$. The resulting magnetic texture is shown in Fig. 2(a). It yields a
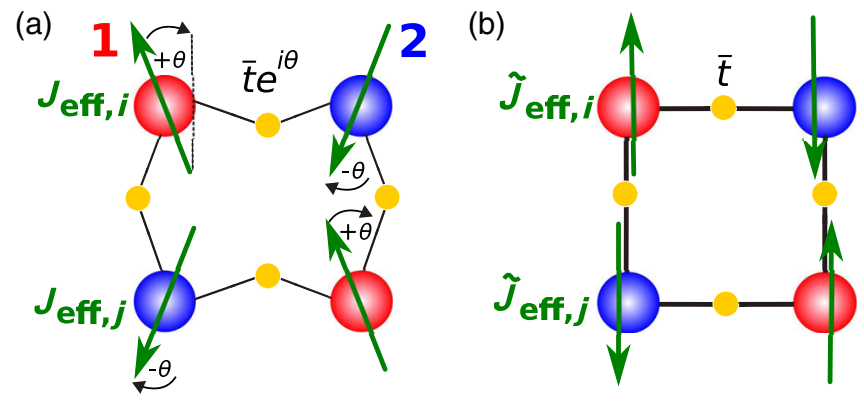

FIG. 2. (a) The canted antiferromagnetic order at the distorted perovskite lattice as it is seen in a single $\mathrm{IrO}_{2}$ layer of $\mathrm{Sr}_{2} \mathrm{IrO}_{4}$. Angle $\theta$ defines deviation of the effective magnetic moment $\mathbf{J}_{\text {eff }}$ from the collinear alignment. Red and blue circles stand for $\mathrm{Ir}^{4+}$ ions belonging to interpenetrating oppositely oriented magnetic sublattices " 1 " and " 2 " magnetic sublattices having magnetic moment denoted by green arrows. Yellow circles schematically represent positions of the oxygen ions. (b) The square bipartite lattice with collinear antiferromagnetic order characterized by rotated magnetic moments $\tilde{\mathbf{J}}_{\text {eff }}$ obtained after the gauge transformation. 
nonzero net moment within a single $\mathrm{IrO}_{2}$ plane. The presented texture due to the canting contrasts with the one described by the Hamiltonian Eq. (1) and depicted in Fig. 1(b). Below we analytically show that this texture can be effectively reduced to a regular collinear antiferromagnetic texture inherent to the Hubbard model.

A single-band Hubbard model for effective total angular momentum $J_{\text {eff }}=1 / 2$ was proposed recently to illustrate the low-energy physics in $\mathrm{Sr}_{2} \mathrm{IrO}_{4}$ [23]. In addition to a renormalized Coulomb repulsion $U_{\text {eff }}$, the model includes nearest-neighbor hopping on a square bipartite lattice with a spin-dependent phase $\phi_{i j}(\sigma, \theta)$ due to the spin-orbit coupling. Here we consider such a Hamiltonian in the presence of a spatially uniform time-dependent electric field:

$$
H(\tau)=-\bar{t} \sum_{\langle i j\rangle, \sigma= \pm} e^{i \psi_{i j}(\tau)} e^{i \phi_{i j}(\sigma, \theta)} c_{i \sigma}^{\dagger} c_{j \sigma}+U_{\text {eff }} \sum_{i} n_{i \uparrow} n_{i \downarrow}
$$

Here, $\bar{t}$ stands for an effective hopping term. The Peierls substitution [24] with the phase factor $\psi_{i j}(\tau)$ is used to account for the electric field which changes its value with time $\tau$. The Peierls phase is given by $\psi_{i j}(\tau)=-q \mathbf{A}(\tau) \cdot\left(\mathbf{r}_{i}-\mathbf{r}_{j}\right)$, where $q$ is the elementary charge and $\mathbf{r}_{i}$ is a coordinate vector of the electron. The phase $\phi_{i j}$ is defined as $\phi_{i j}(\sigma, \theta)=2 \zeta_{i} \theta \sigma$. The structure factor $\zeta_{i}$ defines the magnetic texture. It takes a staggered pattern in this model, so that $\zeta_{i}=+1$ if $i$ belongs to sublattice 1 of the bipartite square lattice and -1 for the sublattice 2 . Consider a unitary transformation:

$$
R=e^{-i \theta \sum_{i \sigma} \sigma \zeta_{i} c_{i \sigma}^{\dagger} c_{i \sigma}}
$$

Under this local rotation we have renormalization of creation and anihilitation operators; e.g., $\tilde{c}_{i \sigma}=R c_{i \sigma} R^{\dagger}=c_{i \sigma} e^{i \sigma \zeta_{i} \theta}$. The transformed Hamiltonian $\tilde{H}$ reads:

$$
\begin{aligned}
\tilde{H}(\tau) & =R H(\tau) R^{\dagger} \\
& =-\bar{t} \sum_{\langle i j\rangle, \sigma= \pm} e^{i \psi_{i j}(\tau)} \tilde{c}_{i \sigma}^{\dagger} \tilde{c}_{j \sigma}+U_{\text {eff }} \sum_{i} \tilde{n}_{i \uparrow} \tilde{n}_{i \downarrow} .
\end{aligned}
$$

It is seen that through a local basis rotation $R$ the Hamiltonian Eq. (2) can be mapped to a regular Hubbard Hamiltonian Eq. (1) with spin-independent hopping, i.e., without spin-orbit coupling and with compensated collinear antiferromagnetic order. Note that this is only possible if the provided magnetic texture results in zero total phase change as the electron hops around a closed loop, such as a staggered pattern in $\mathrm{Sr}_{2} \mathrm{IrO}_{4}$ [see Fig. 2(a)].

The Cartesian components of the effective angular moment can be defined as $J_{\text {eff }}^{\mu}=\frac{1}{2} \sum_{\alpha, \beta= \pm} c_{\alpha}^{\dagger} \sigma_{\alpha \beta}^{\mu} c_{\beta}$. Here, $\sigma_{\alpha \beta}^{\mu}$ are components of Pauli matrices and $\mu=x, y, z$. Specifically, the in-plane $x$ and $y$ components read:

$$
\left(\begin{array}{c}
J_{\text {eff }}^{x} \\
J_{\text {eff }}^{y}
\end{array}\right)=\frac{1}{2}\left(\begin{array}{c}
c_{\downarrow}^{\dagger} c_{\uparrow}+c_{\uparrow}^{\dagger} c_{\downarrow} \\
i\left(c_{\downarrow}^{\dagger} c_{\uparrow}-c_{\uparrow}^{\dagger} c_{\downarrow}\right)
\end{array}\right)
$$

The magnetic components under the unitary transformation experience rotation with respect to the $z$ direction, such that components of the transformed angular momentum $\tilde{J}_{\text {eff }}$ are given by

$$
\left(\begin{array}{c}
\tilde{J}_{\mathrm{eff}, i}^{x} \\
\tilde{J}_{\mathrm{eff}, i}^{y}
\end{array}\right)=\left(\begin{array}{c}
R J_{\mathrm{eff}, i}^{x} R^{\dagger} \\
R J_{\mathrm{eff}, i}^{y} R^{\dagger}
\end{array}\right)=\left(\begin{array}{cc}
\cos \theta & -\zeta_{i} \sin \theta \\
\zeta_{i} \sin \theta & \cos \theta
\end{array}\right)\left(\begin{array}{c}
J_{\mathrm{eff}, i}^{x} \\
J_{\mathrm{eff}, i}^{y}
\end{array}\right) .
$$

This relation holds for any time $\tau$ because $\tilde{J}_{\text {eff }}^{\mu}(\tau)=R J_{\text {eff }}^{\mu}(\tau) R^{\dagger}$. The latter can be seen from the fact that $\tilde{J}_{\text {eff }}^{\mu}(\tau)$ and $R J_{\text {eff }}^{\mu}(\tau) R^{\dagger}$ are equal in equilibrium $(\tau<0)$ and satisfy the same Heisenberg equation of motion:

$i \frac{d}{d \tau} R J_{\mathrm{eff}}^{\mu}(\tau) R^{\dagger}=R\left[J_{\mathrm{eff}}^{\mu}(\tau), H(\tau)\right] R^{\dagger}=\left[R J_{\mathrm{eff}}^{\mu}(\tau) R^{\dagger}, \tilde{H}(\tau)\right]$.

In general, one can assume that collinear spin moments $\tilde{\boldsymbol{J}}_{\text {eff }, i}$ align in the $y$ direction in the transformed system so that $\left\langle\tilde{J}_{\mathrm{eff}, i}^{x}(\tau)\right\rangle=0$ and $\left\langle\tilde{J}_{\text {eff }, i}^{y}(\tau)\right\rangle=\zeta_{i} J(\tau)$ with magnitude $J(\tau)$. Solving Eq. (6) yields $\left\langle J_{\text {eff }, i}^{x}(\tau)\right\rangle=J(\tau) \sin \theta$ and $\left\langle J_{\text {eff }, i}^{y}(\tau)\right\rangle=\zeta_{i} J(\tau) \cos \theta$. Thus the canting angle $\theta$ remains intact within the course of dynamics triggered by the external electric field. This remains valid as long as the geometrical structure of the hopping matrix elements is such that electron hopping around a closed loop acquires zero phase change; i.e., the unitary transformation $R$ is possible. As photoexcitation of Mott-Hubbard systems is primarily governed by photoinduced charge transfer which leaves the geometrical structure unaffected, we assume that the canting angle $2 \theta$ between adjacent antiferromagnetic sublattices remains constant even during the course of photodoping. Hence, the net magnetization which originates from the canting can serve as a direct measure of the underlying antiferromagnetic order even out of the equilibrium.

\section{STRUCTURAL CHARACTERIZATION OF THE SAMPLE}

$\mathrm{Sr}_{2} \mathrm{IrO}_{4}$ thin films were deposited on commercially available $\mathrm{TiO}_{2}$-terminated (001) $\mathrm{SrTiO}_{3}$ (STO) substrates (CrysTec $\mathrm{GmbH}$ ) by pulsed laser deposition using a $\mathrm{KrF}$ excimer laser $(248 \mathrm{~nm})$. The substrate was mounted with two clamps and heated by an infrared laser to $920^{\circ} \mathrm{C}$, as measured with an optical pyrometer. A laser fluence of $1.1 \mathrm{~J} / \mathrm{cm}^{2}$ and an oxygen partial pressure of $0.6 \mathrm{mbar}$ were used. The growth was monitored by in situ reflection high-energy electron diffraction (RHEED). To prevent degradation, the film was capped with 10 unit cells (u.c.) of STO deposited at $600^{\circ} \mathrm{C}$ 

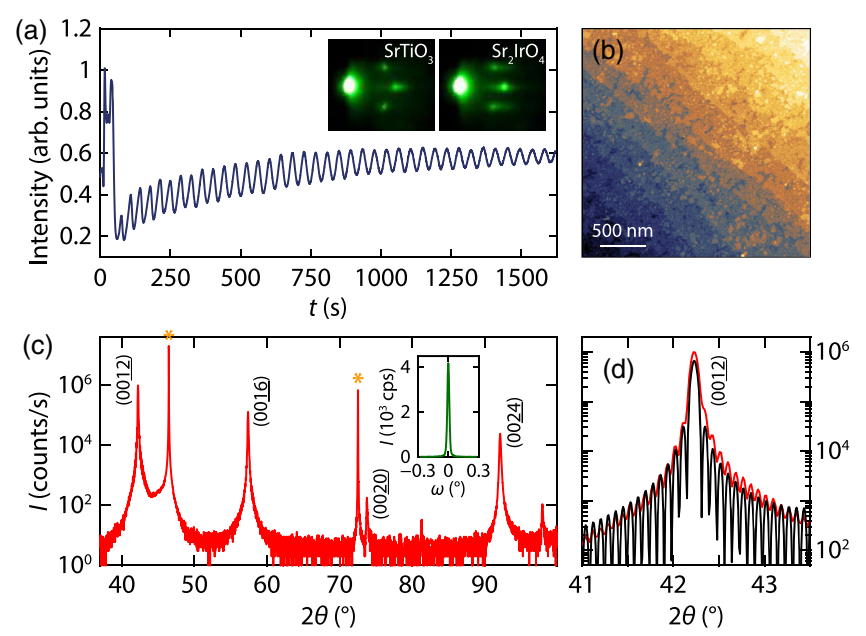

FIG. 3. (a) RHEED intensity oscillations of the specular spot during the growth of a 45-u.c. $\mathrm{Sr}_{2} \mathrm{IrO}_{4}$ film. Four oscillations correspond to the growth of 1-u.c. $\mathrm{Sr}_{2} \mathrm{IrO}_{4}$. (b) Atomic force microscopy topographic image of the surface of a 10-u.c. $\mathrm{Sr}_{2} \mathrm{IrO}_{4}$ film. (c) X-ray diffraction scan of STO capped 45-u.c. $\mathrm{Sr}_{2} \mathrm{IrO}_{4}$ film. Inset: Rocking curve around the $\mathrm{Sr}_{2} \mathrm{IrO}_{4}(00 \underline{12})$ reflection (d). X-ray diffraction scan around the $(0012)$ reflection. The solid black line is a simulation of the diffracted intensity with 45 u.c. and $c=25.66 \AA$.

under the same fluence and oxygen partial pressure. After growth, the sample was annealed for $1 \mathrm{~h}$ at $600^{\circ} \mathrm{C}$ in 300 mbar $\mathrm{O}_{2}$ to compensate for possible oxygen deficiency.

Figure 3(a) shows the RHEED intensity of the specular spot during the $\mathrm{Sr}_{2} \mathrm{IrO}_{4}$ growth. The persistent intensity oscillations indicate a layer-by-layer growth mode, which enabled us to precisely control the thickness of the film to 45 u.c. An atomic force microscopy image of the surface of an uncapped $\mathrm{Sr}_{2} \mathrm{IrO}_{4}$ film shows a step-and-terrace structure, confirming the layer-by-layer growth mode and showing that the surface is atomically smooth [see Fig. 3(b)]. The crystal structure of the 45-u.c. film was studied by x-ray diffraction. Figure 3(c) shows a $2 \theta-\omega$ measurement, showing that no other crystalline phases are present. The clear finite size oscillations and the small full width at half maximum $\left(0.024^{\circ}\right)$ of the rocking curve (see inset) evidence long-range crystalline order. Figure 3(d) shows the $\mathrm{Sr}_{2} \mathrm{IrO}_{4}(0012)$ reflection together with a simulation of the diffracted intensity for a thickness of 45 u.c. and a $c$-axis parameter of $25.66 \AA$. The thickness is in good agreement with the thickness determined by RHEED. The $c$ axis is slightly smaller than that of bulk $\mathrm{Sr}_{2} \mathrm{IrO}_{4}(25.79 \AA)$, which results from the tensile strain $(-0.7 \%$ lattice mismatch) from the STO substrate. Additionally, reciprocal space maps (not shown here) show that the film is coherently strained to the substrate.

\section{MAGNETIC AND MAGNETO-OPTICAL CHARACTERIZATION OF THE SAMPLE}

The complex magnetic structure of $\mathrm{Sr}_{2} \mathrm{IrO}_{4}$ can be effectively reduced to a pair of interpenetrating antiferromagnetic sublattices, characterized by the magnetizations $\mathbf{M}_{1}$ and $\mathbf{M}_{2}$. The magnetizations are oriented in the (001) plane such that they are not exactly antiparallel but canted by some angle $\theta$. The value of the net magnetic moment was determined measuring the net magnetization $M$ employing a SQUID magnetometer. Figure 4(b) shows the temperature dependence of the magnetic moment while cooling in a magnetic field of $50 \mathrm{mT}$ applied in the film plane. At a temperature of $100 \mathrm{~K}$, the magnetic moment in the film plane was measured to be $30 \times 10^{-3} \mu_{B}$ per Ir atom, in good agreement with Refs. [25,26]. The temperature dependence indicates a Néel temperature $T_{N} \sim 190 \mathrm{~K}$, which is slightly lower than the $T_{N}=240 \mathrm{~K}$ of bulk
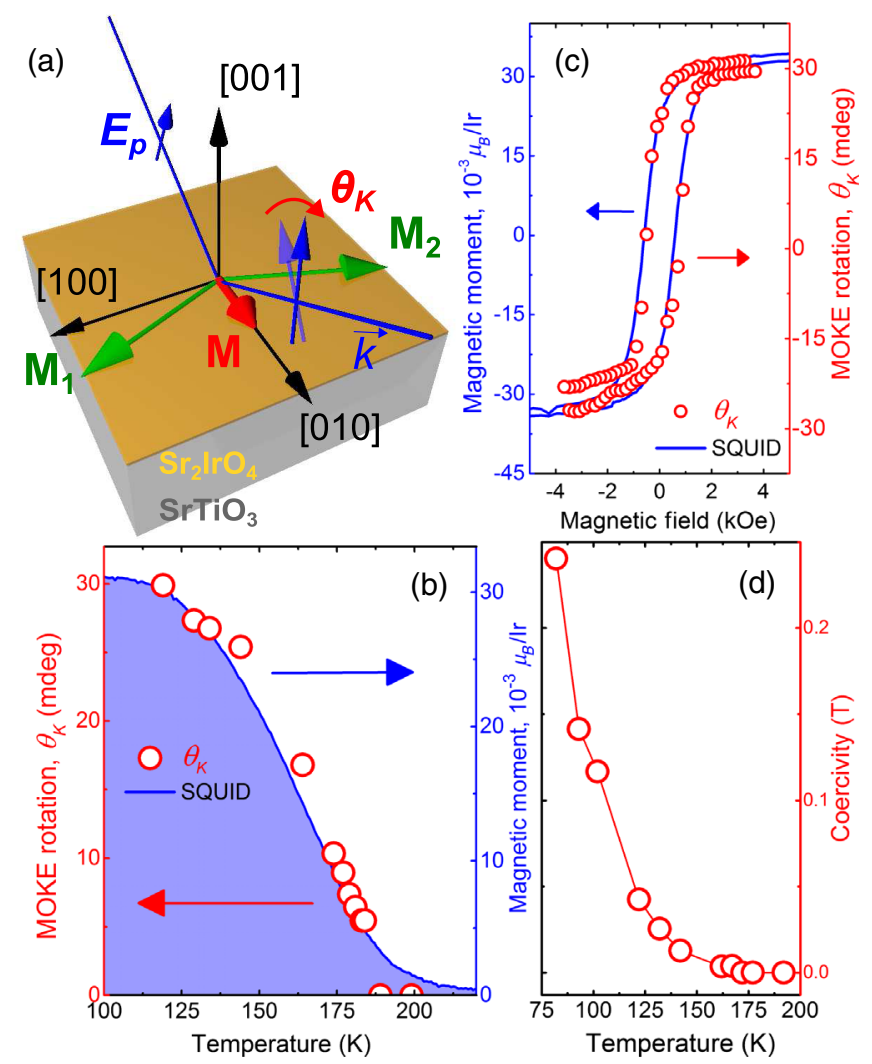

FIG. 4. (a) Experimental geometry employed for the longitudinal MOKE experiment. The blue arrows depict optical path and orientation of the electric field $E_{p}$ of the incident light. $\theta_{K}$ is the rotation of the polarization plane due to the MOKE. (b) Left axis (blue line): $\mathrm{Sr}_{2} \mathrm{IrO}_{4}$ magnetic moment per $\mathrm{Ir}$ atom as a function of the external magnetic field applied in the sample plane. The data are acquired with a SQUID magnetometer. Right axis (red circles): The MOKE rotation as a function of the external magnetic field applied in the sample plane. The incident angle is close to $30 \mathrm{deg}$. The data are measured at $120 \mathrm{~K}$. (c) Left axis (red circles): The amplitude of the MOKE signal measured for various temperatures. Right axis (blue line): Temperature dependence of magnetization per Ir atom acquired with a SQUID. The data are acquired during cooling down with a field of $0.5 \mathrm{~T}$ applied in the sample plane. (d) Temperature dependence of the coercive field. 
$\mathrm{Sr}_{2} \mathrm{IrO}_{4}$ [27]. We attribute the reduction of $T_{N}$ to the epitaxial strain supplied by the substrate, which modifies octahedral rotation angles and hence affects the strength of the magnetic order in the thin film limit. Effects of the strain on the Néel temperature of $\mathrm{Sr}_{2} \mathrm{IrO}_{4}$ were discussed earlier in Refs. [26,28]. The magnetic hysteresis loops were measured after cooling in $0.5 \mathrm{~T}$, as exemplified in Fig. 4(c). Contrary to measurements on bulk $\mathrm{Sr}_{2} \mathrm{IrO}_{4}$, we observed no sign of a metamagnetic phase transition, as reported in Refs. [27,29]. We note that measurements with a magnetic field applied along the sample normal reveal no out-ofplane magnetization component.

For static magneto-optical characterization, we employed longitudinal MOKE [17] in the configuration shown in Fig. 4(a). Linearly polarized light at a wavelength of $400 \mathrm{~nm}$ was incident on the sample at an angle close to $30^{\circ}$ with respect to the sample normal. The MOKE results in a rotation $\theta_{K}$ of the polarization plane of the reflected light, such that $\theta_{K} \propto(\mathbf{k}, \mathbf{M})$. Here, $\mathbf{k}$ is a unit vector along the propagation direction and $\mathbf{M}$ is the net magnetization. The changes in the polarization of the reflected light were measured using a Wollaston prism, which splits light into two orthogonally polarized components. The difference in intensity of these two beams proportional to the polarization rotation was measured with the help of a pair of balanced photodiodes. The probe wavelength of $400 \mathrm{~nm}$ corresponds to a photon energy of $3.1 \mathrm{eV}$ and matches the charge-transfer transition from O $2 p$ to Ir $5 d$ orbitals [see Fig. 5(a)]. This transition is a genuine absorption feature of many transition-metal oxides and is responsible for their magneto-optical effects [30-32]. Magnetic fields up to $0.5 \mathrm{~T}$ were produced by a conventional electromagnet and the sample temperature was varied in a continuous flow cryostat. The rotation obtained as a function of the magnetic field is depicted in Fig. 4(c), which shows a clear hysteretic response. The value of the Kerr rotation is substantial, reaching nearly $30 \mathrm{mdeg}$ at low temperature. Such a strong magneto-optical response can be attributed to the large value of the spin-orbit coupling. Figure 4(b) shows that the MOKE rotation vanishes for temperatures above $190 \mathrm{~K}$, agreeing well with the Néel temperature $T_{N}$ obtained from SQUID measurements.

\section{ULTRAFAST PHOTODOPING OF $\mathrm{Sr}_{2} \mathrm{IrO}_{4}$ THIN FILMS}

The net magnetization of $\mathrm{Sr}_{2} \mathrm{IrO}_{4}$ in combination with the substantial value of the MOKE rotation offers unique opportunity to explore ultrafast magnetism in a Mott insulator by photodoping.

For the experiment we employed a Ti:sapphire amplified laser system (Spectra-Physics, Spitfire), producing pulses at a central wavelength of $800 \mathrm{~nm}(1.55 \mathrm{eV})$ and operating at $1-\mathrm{kHz}$ repetition rate. The pump was focused under normal incidence onto a spot with a full width at half maximum of nearly $800 \mu \mathrm{m}$. The duration of the pump

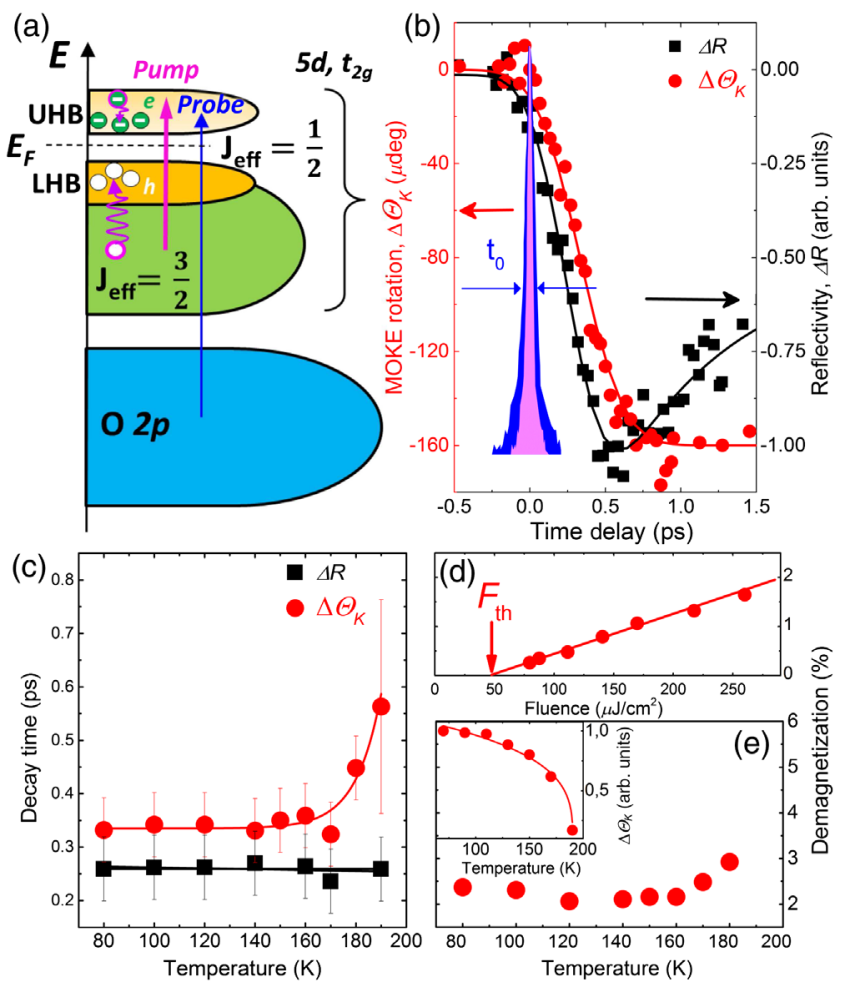

FIG. 5. (a) Schematic band structure of $\mathrm{Sr}_{2} \mathrm{IrO}_{4}$. The pink arrow indicates the optical transition launched by the pump pulse with a photon energy of $1.55 \mathrm{eV}$. Open circles and circles with a dash indicate photoinduced holes $h$ and electrons $e$, respectively. Pink spring illustrates the process of the impact ionization and relaxation of the photoinduced carriers to the bottom of the UHB and LHB. The solid blue line denotes charge-transfer transition between oxygen $(2 p)$ and iridium ion $(5 d)$ probed with the probe pulse having a photon energy of $3.1 \mathrm{eV}$. (b) Left axis: Time-resolved transient MOKE signal triggered by the pump pulse. Right axis: Time-resolved transient reflectivity signal triggered by the pump pulse. Solid lines are fits to the data. The fits are represented by an exponential decay function multiplied by the error function. Sample temperature is $80 \mathrm{~K}$ and the pump fluence is $100 \mu \mathrm{J} / \mathrm{cm}^{2}$. The blue and violet lines show the autocorrelation functions of the probe and pump pulses, respectively. (c) Temperature dependence of the magnetization decay time (red circles) and time of the fast rising edge in the reflectivity signal (black squares). (d) Value of the pump-induced demagnetization as a function of the pump fluence. (e) Value of the pump-induced demagnetization as a function of the sample temperature. The inset shows the magnitude of the transient pump-induced MOKE rotation $\Delta \theta_{K}$ as a function of the sample temperature. The fluence of the pump pulse is $250 \mu \mathrm{J} / \mathrm{cm}^{2}$.

pulse measured just before the sample was nearly $60 \mathrm{fs}$. As shown in Fig. 5(a), the photons at the pump energy excite electrons from the occupied $J_{\text {eff }}=3 / 2$ and $J_{\text {eff }}=1 / 2$ bands to the unoccupied $J_{\text {eff }}=1 / 2$ band $[28,33,34]$. The probe pulse having a central wavelength of $400 \mathrm{~nm}$ was generated using $0.5 \mathrm{~mm}$ of a nonlinear $\beta$-barium-borate crystal as the second harmonic of the seeding pulse. The duration of the probe pulse was $80 \mathrm{fs}$, as estimated by the 
method described in Ref. [35]. Both the pump and probe beams were focused on the same spot and the required time resolution was obtained by varying the optical path length for one of the beams with the help of a precision mechanical delay line. The angle of incidence of the probe beam was chosen to be the same as the one employed for the static MOKE. In order to satisfy pulse-to-pulse repeatability of the stroboscopic measurements, an external magnetic field at a value slightly surpassing the coercive field [for the values, see Fig. 4(d)] was applied in the sample plane. In the experiment, the transient dynamics of the MOKE signal $\Delta \theta_{K}$ and the reflectivity $\Delta R$ of the probe pulse were detected simultaneously.

Figure 5(b) shows transient time-resolved dynamics of the pump-induced rotation of the polarization plane of the probe pulse $\Delta \theta_{K}$ together with the transient dynamics of the reflectivity $\Delta R$. The pump pulse initiates fast changes in both signals on a subpicosecond timescale. As the penetration depth of the probe pulse is around $70 \mathrm{~nm}$ [33], which is significantly smaller than the thickness of the film, the transient dynamics originates solely from the pumpinduced changes in the film and not from the substrate. The rotation dynamics is predominantly an odd function of the applied field while the reflectivity shows no sensitivity on the sign of the magnetic field. To separate $\Delta \theta_{K}$ from other phenomena of nonmagnetic origin which also may result in a rotation of the polarization plane (e.g., photoinduced birefringence), we calculated the difference of the polarization rotation measured at two magnetic fields of opposite polarities. Thus, the sudden change in $\Delta \theta_{K}$ can be attributed to the ultrafast loss of the magnetization, i.e., demagnetization. The ultrafast dynamics seen in the $\Delta R$ signal can be attributed to the dynamics of the photoinduced carriers: holons and doublons. The fact that the magnetization dynamics is slower than the carrier dynamics and duration of the pump pulse itself indicates the irrelevance of an instantaneous light-induced transfer of angular momentum, originating from optical transitions between spin-orbital states with $J_{\text {eff }}=3 / 2$ and $J_{\text {eff }}=1 / 2$ [36,37]. The observed dynamics can be attributed rather to photodoping of the upper (UHB) and the lower Hubbard bands (LHB), similar to experiments reported in Refs. [15,16, 38-41]. Here we assume that the observed ultrafast demagnetization reveals the dynamics of the melting of the underlying antiferromagnetic order. The assumption is theoretically discussed in Sec. II and experimentally supported by the fact that $\Delta \theta_{K}$ similarly to $\theta_{K}$ shows critical temperature behavior approaching $T_{N}$; see inset in Fig. 5(e). As shown in Fig. 5(c), the time required to melt magnetic order is consistently longer than the one seen in the transient reflectivity. Moreover, it displays a pronounced upturn in the vicinity of the Néel temperature, while the decay time in the reflectivity channel shows no visible temperature dependence. The melting time far from $T_{N}$ is $0.34 \pm 0.06 \mathrm{ps}$ and nearly matches the upper estimate of the decay time of the 3D magnetic order, implying a destruction of the intra- and interplane magnetic correlations, as reported in Ref. [15].

Interestingly, the demagnetization as a function of the pump intensity reveals a threshold fluence $F_{\text {th }}=40 \mu \mathrm{J} / \mathrm{cm}^{2}$ [Fig. 5(d)], which corresponds to the absorption of nearly $10^{-3}$ photons per Ir atom. The threshold fluence can be caused by localization of small concentrations of the holondoublon pairs due to polaronic effects [41]. The absolute values of the demagnetization $\Delta \theta_{K} / \theta_{K}$ do not show any pronounced temperature dependence [see Fig. 5(e)]. All the measurements reported here were done in the regime where the pump-induced signal scales linearly with the pump fluence, as is shown in Fig. 5(d).

The nonequilibrium DMFT was shown to be rewarding in describing an early stage in the evolution of the antiferromagnetic order after the ultrafast photodoping [11]. To simulate the photoinduced dynamics in $\mathrm{Sr}_{2} \mathrm{IrO}_{4}$, we exploit the fact that the Hamiltonian Eq. (2) can be unambiguously mapped to a regular Hubbard Hamiltonian Eq. (1), and for this reason we consider a minimal one-band Hubbard model on a Bethe lattice using the nonequilibrium DMFT approach. In the effective model, we pick up the parameters $U=0.5 \mathrm{eV}$ and bandwidth $W=0.4 \mathrm{eV}$ from Ref. [21], thus $U / W \sim 1.3$, resulting in the Néel temperature of about $195 \mathrm{~K}$ [see inset of Fig. 6(b)]. At time zero the system is disturbed by a short electric pulse with the
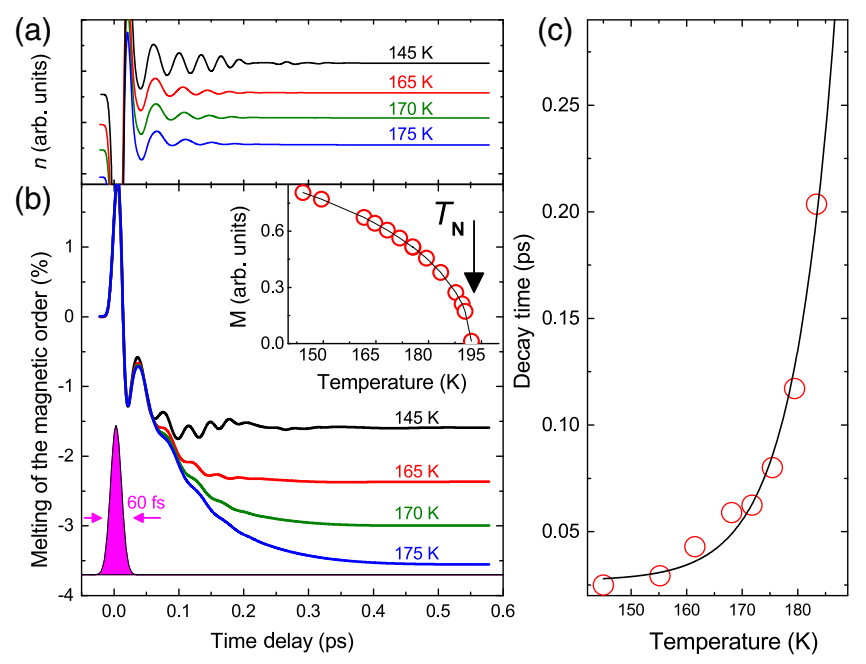

FIG. 6. (a) The nonequilibrium DMFT simulations of the lightinduced dynamics of the nonequilibrium population of doublons and holons $n$ for various temperatures. The excitation is caused by the ultrashort pulse having the duration of $60 \mathrm{fs}$. (b) The corresponding light-induced magnetic dynamics in $\mathrm{Sr}_{2} \mathrm{IrO}_{4}$ for the same temperatures. The inset shows the temperature dependence of the net magnetization obtained from the Hubbard model solved on the Bethe lattice. (c) The exponential decay time of the magnetic order as a function of the temperature extracted from the time traces obtained within the DMFT simulations. The solid black line is a guide to the eye. 
duration approximately equal to $60 \mathrm{fs}$, to simulate the photoexcitation in the experiment. Figure 6(a) shows that the action of the electric pulse leads to the nearly instantaneous emergence of long-living population of the holons and doublons having concentration $n$. In agreement with the experiment, the DMFT simulations demonstrate nearly exponential dynamics of the magnetic melting with a substantial slowing-down in the vicinity of the $T_{N}$; see Figs. 6(b) and 6(c). Despite the qualitative similarities, it can be seen that the course of the demagnetization in our experiment proceeds on significantly longer time; for comparison, see Figs. 6(c) and 5(c). Moreover, although the simulations suggest large enhancement of the degree of the melting when the temperature approaches $T_{N}$ [see Fig. 6(b)], the experiment shows that the demagnetization amplitude is weakly sensitive to the temperature [see Fig. 5(e)].

Our experiment shows that every photon on average results in the destruction of nearly five magnetic moments. This implies that every photon contributes to multiple hopping processes. In our experiment, the initial photoexcited states have a large excess energy as the pump photon energy is much larger than the Mott gap. This might lead to the excitation of other carriers in the $J_{\text {eff }}=3 / 2$ band via the impact ionization that subsequently contributes to the changes in reflectivity and magnetization $[42,43]$. Hence, the decay times seen in our experiment are associated with higher-order scattering processes and can thus be related to the average time necessary to accomplish the processes of the impact ionization. Naturally, these times can be significantly longer than the fast times seen in the DMFT simulations considering the effect of only one band.

Overall, the nonequilibrium DMFT simulations confirm the leading role of photogenerated electron-hole pairs in the subpicosecond melting of the magnetic order, but they cannot reproduce the experimentally observed dependencies even qualitatively. This discrepancy calls for even more complex DMFT calculations that are technically very challenging and thus call for exemplified phenomenological models.

Figure 7(a) shows the time-resolved dynamics of the Kerr rotation on a longer timescale. From these data it can be seen that the subpicosecond magnetization quench is followed by a slower recovery. The recovery dynamics reveals two relaxation times: the fast recovery happens on a sub-10-ps timescale and restores the major part of the magnetization, while on longer timescales a further slow remagnetization finalizes the recovery process. We attribute the latter to the diffusion of excess heat from the irradiated area [44]. To study the origin of the first relaxation process, we measured the time-resolved dynamics of the magnetization at different temperatures [see Fig. 7(b)]. Even though the decay of magnetic order and electronic perturbation happen on similar timescales, their recovery times at elevated
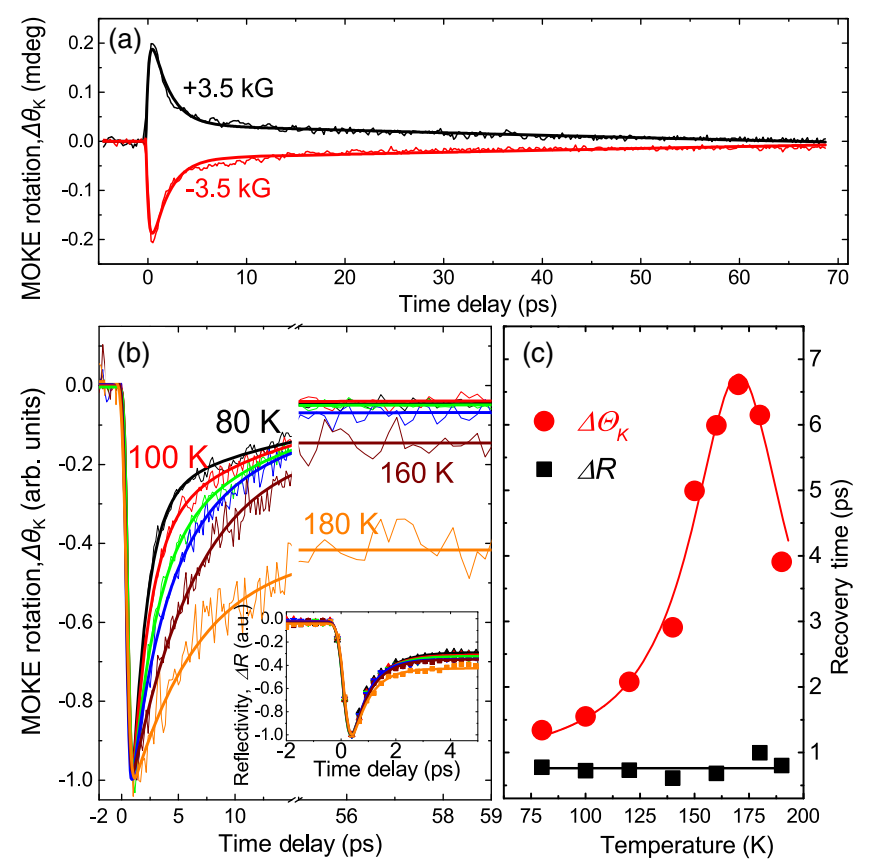

FIG. 7. (a) Time-resolved changes in the MOKE rotation caused by the pump pulse as a function of time delay between the pump and the probe pulses. The data are shown for two opposite values of external magnetic field $\pm 3.5 \mathrm{kOe}$. The sample temperature is $80 \mathrm{~K}$ and the pump fluence is $100 \mu \mathrm{J} / \mathrm{cm}^{2}$. (b) Transient changes in the MOKE rotation as a function of time delay between the pump and probe pulses measured at various temperatures. The time-resolved traces are normalized to the maximal value to emphasize the recovery dynamics. The inset shows the corresponding changes in the reflectivity signal. (c) The recovery time for the magnetization (red circles) and reflectivity signal (black squares) as a function of the sample temperature. The solid lines are guides to the eye.

temperatures are markedly different [see Fig. 7(c)]. The recovery dynamics of the magnetization shows a pronounced slowing-down in the vicinity of the Néel temperature, whereas the relaxation observed in the reflectivity signal remains nearly temperature independent. Only at low temperatures the time required for the magnetization to recover starts to approach the recovery time seen in the reflectivity signal. In Ref. [15] it was demonstrated that the recovery of the charge and in-plane magnetic order proceed on a similar timescale. Our work demonstrates that this is true only when the system is far from the magnetic phase transition. The magnetization recovery time peaks nearly at $175 \mathrm{~K}$, i.e., slightly below $T_{N}=190 \mathrm{~K}$ [see Fig. 7(c)]. It suggests that, upon completion of the ultrafast demagnetization, the temperature of the laser-excited spot is about $15 \mathrm{~K}$ above the surrounding heat bath. As the experiment is performed at low repetition rate and applied fluences are low, a steady-state temperature increase via cumulative laser heating can be excluded. A similar temperature shift of the relaxation anomaly to temperatures lower than the Néel point was reported in Ref. [45] where it was attributed to fast 
equilibration of the spin and lattice degrees of freedom. The short time, seen in our experiment, can be related to the enhanced strength of the spin-orbit coupling in $\mathrm{Sr}_{2} \mathrm{IrO}_{4}$.

\section{PHENOMENOLOGICAL DESCRIPTION OF THE ULTRAFAST DYNAMICS USING ONSAGER'S FORMALISM}

To account for the dynamics caused by the impact ionization in the DMFT simulations, a model that includes multiple bands with different bandwidth has to be considered. Such a task represents technically a very complex computational problem. In contrast, the phenomenological models, although being an oversimplified description of physical processes, usually are easily solvable and allow one to highlight underlying physical mechanisms. Here we suggest a phenomenological model based on Onsager's formalism [46], which describes the nonequilibrium dynamics of a Mott insulator and effectively accounts for the impact ionization processes.

The spontaneous magnetization in a weak ferromagnet is given by the expression

$$
\mathbf{M}=\frac{1}{2} g_{J} l \mathbf{J}_{\mathrm{eff}},
$$

where $l$ stands for the concentration of magnetic moments, $\mathbf{J}_{\text {eff }}=\mathbf{J}_{\text {eff,1 }}+\mathbf{J}_{\text {eff,2 }}$ is the net magnetic moment for each pair of magnetic ions, and $g_{J}$ is the Landé factor. From here on we use for denomination of the $\mathbf{J}_{\text {eff }}$ a shortening, $\mathbf{J}$. Previously, models of experimental studies of ultrafast magnetism, such as atomistic spin dynamics simulations and the $3 T$ model, assumed $l$ to be given by the concentration of magnetic ions and to be constant. Furthermore, the decay of the magnetic moment is to be solely governed by the disorder of the local moments with respect to the collinear ground state [5]. Therefore, in such a case the magnetization dynamics is solely driven by the dynamics of $J$. For a magnet brought out of equilibrium the value of the net magnetic moment $J$ changes only as a result of the interactions between microscopic spins as described phenomenologically by the dependence of the free energy on magnetization [47]. In the Mott insulators, the concentration of magnetic moments is tightly related to their onsite localization. Photodoping causes delocalization of the electrons and subsequent transfer of the magnetic moment between the magnetic sublattices, accompanied by a depletion of the magnetic moments in the volume. This implies that in Mott insulators with weak ferromagnetism, contrary to conventional magnets, the variation of $M$ is given not only by a variation of $J$ but also by $l$, such that for small values of $\Delta l$ and $\Delta J$, it reads:

$$
\frac{\Delta M}{M}=\left(\frac{\Delta l}{l}+\frac{\Delta J}{J}\right)
$$

If $n$ denotes the concentration of the pairs, then the reduction in the concentration of the localized moments is given by

$$
\Delta l=-2 n .
$$

The Onsager equations for a nonequilibrium concentration of the holons and doublons $n$ and uncompensated magnetic moment $J$ read:

$$
\frac{d n}{d \tau}=-\eta_{n} \frac{\delta \mathcal{F}(n, J)}{\delta n}, \quad \frac{d J}{d \tau}=-\eta_{m} \frac{\delta \mathcal{F}(n, J)}{\delta J} .
$$

Here, the constants $\eta_{n}$ and $\eta_{m}$ are phenomenological parameters describing the relaxation of the photoinduced carriers and the magnetic moments in time $\tau$, respectively. The phenomenological form of the free energy $\mathcal{F}$ is chosen as

$\mathcal{F}(n, J)=\frac{1}{2} \alpha n^{2}+\frac{1}{4} \beta\left(J^{2}-J_{0}^{2}\right)^{2}+\frac{1}{2} \gamma n\left(J^{2}-J_{0}^{2}\right)-f(\tau) n$,

where the first term describes the excess energy of the holons and doublons. The second term stands for the magnetic potential in the form of Landau's expansion [see Fig. 8(a)]. $J_{0}$ is the equilibrium value of the net moment for a given temperature and $n=0$. The third term phenomenologically describes the coupling between the photoexcited quasiparticles and the magnetic moments. The last term describes the excitation of the quasiparticles by a light pulse having a Gaussian time profile $f(\tau)=f_{0} e^{-\tau^{2} / 2 \tau_{p}^{2}}$, where $f_{0}$ is a quantity proportional to the laser fluence and $\tau_{p}$ is the duration of the excitation pulse. $\alpha, \beta$, and $\gamma$ are phenomenological parameters. The combination of these parameters with $\eta_{n}$ and $\eta_{m}$ gives experimentally measured values, such as decay and recovery times. In Eq. (12) the terms containing spatial gradients of the net moment $\mathbf{J}$ are intentionally omitted, as the area excited and probed in the experiment is significantly larger than the length scale of the magnetic inhomogeneity. The latter originates from the emergence of the holons which represent dangling exchange bonds and are expected to not exceed a couple of intersite distances.

The presence of the nonequilibrium concentration of the holons and doublons results not only in a depletion of the number of localized magnetic moments, but also in a reduction of the net moment according to

$$
J^{2}(n)=J_{0}^{2}-\frac{\gamma}{\beta} n .
$$

The coupled equations Eq. (11) enable us to analyze the relaxation dynamics of both the carriers and the net magnetic moment. To simplify the problem we neglect 
(a)
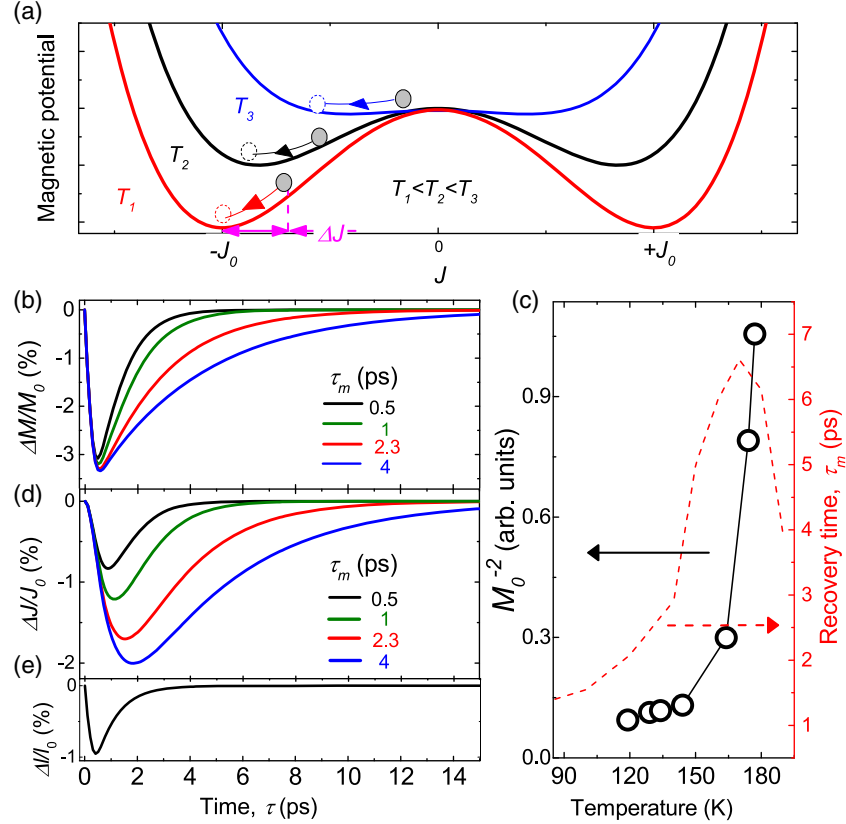

FIG. 8. (a) Profile of the magnetic free energy in the form of the Landau expansion containing terms up to fourth order, shown for various temperatures below the Néel point. (b) Numeric simulation of the light-induced magnetization dynamics. The normalized value $\Delta M / M_{0}$ is plotted. (c) Black hollow dots: square of the inverse magnetization $M_{0}^{-2}$ plotted as a function of the temperature. Red dashed line: recovery time of the magnetization as a function of temperature. The same data are presented in Fig. 7(c). The data are shown for comparison. (d) Numeric simulation of the light-induced dynamics of the net magnetic moment. The normalized value $\Delta J / J_{0}$ is plotted. (e) Numeric simulation of the light-induced changes in the concentration of the localized moments. The normalized value $\Delta l / l_{0}$ is plotted.

an action of the magnetic moments on dynamics of the quasiparticles and consider only the influence of the photocarriers on $J$. Accounting for the inverse effect does not significantly alter the results. In this approximation, one obtains

$$
\frac{d n}{d \tau}=-\frac{1}{\tau_{c}} n+F(\tau)
$$

where $\tau_{c}=\left(\alpha \eta_{n}\right)^{-1}$ defines the decay time of the photoexcited carriers and $F(\tau)=\eta_{n} f(\tau)$. The solution of Eq. (14) is given by

$$
n(\tau)=\eta_{n} e^{-\tau / \tau_{c}} \int_{0}^{\tau} f(\zeta) e^{\zeta / \tau_{c}} d \zeta
$$

From this it follows that the holons and doublons are produced by the Gaussian-shaped light pulse within a characteristic time $\tau_{p}$ and relax back to equilibrium exponentially, with the time $\tau_{c}$, which we attribute to the recovery time seen in transient reflectivity.
After substituting the solution Eq. (15) in the relaxation equation for the net moment, one obtains

$$
\frac{d J}{d \tau}=-\frac{1}{\tau_{0}} J\left(J^{2}-J_{0}^{2}\right)-\gamma \eta_{m} J n(\tau)
$$

where $\tau_{0}=\left(\beta \eta_{m}\right)^{-1}$. Since in the experiment $\Delta M \ll M_{0}$, Eq. (16) can be linearized. Introducing $j=\Delta J / J_{0}$, it reads:

$$
\frac{d j}{d \tau}=-\frac{1}{\tau_{m}} j-\gamma \eta_{m} n(\tau),
$$

where $\tau_{m}=\tau_{0} / 2 J_{0}^{2}$ is related to the recovery of the magnetic order and, being proportional to $\beta^{-1}$, is defined purely by the magnetic potential. As the concentration of the photoexcited carriers stands in the right-hand side of Eq. (17), it implies that the decay of the net moment occurs not only during the emergence of the carriers but all along the relaxation, in agreement with Ref. [9]. As in the experiment $\tau_{m}>\tau_{c}>\tau_{p}$, the solution of Eq. (17) for $\tau \gg\left(\tau_{c}, \tau_{p}\right)$ is given by

$$
j(\tau)=-\gamma \eta_{m} \tau_{m} n_{0} e^{-\tau / \tau_{m}} .
$$

Here, $n_{0} \cong \eta_{n} f_{0} \tau_{p}$ denotes the initial concentration of the nonequilibrium carriers. It is visible that the recovery time of the net moment is defined by the value of $\tau_{m}$, which is inversely proportional to the value of the static magnetization $M_{0}^{-2}(T)$. This result agrees well with the phenomenological Landau-Khalatnikov theory describing relaxation of an order parameter in the vicinity of a second-order phase transition [48].

To account for the impact ionization caused by the multiparticle scattering, the actual duration of the pump pulse was substituted with the lengthened effective value having the duration of $\tau_{p}=0.25 \mathrm{ps}$. The decay time of the carriers was set to the value close to the one seen in the reflectivity signal $\tau_{c}=0.8 \mathrm{ps}$. Figure 8 (b) shows the magnetization dynamics given by the solutions of Eqs. (14) and (17), using Eqs. (9) and (10). The other two values necessary for the simulations, $\eta_{n} f_{0}=0.056$ and $\gamma \eta_{m}=2$, were selected empirically to provide a good match with the experiment. As is seen from Fig. 8(b), overall good qualitative agreement between the experiment and the simulation is achieved. Figure 8(c) shows a comparison of the temperature dependencies for the experimentally determined recovery times and the $M_{0}^{-2}(T)$ values determined from the static MOKE measurements. Both follow a similar trend and diverge upon approaching the Néel temperature, which indicates that the recovery dynamics originates from interactions between microscopic magnetic moments as described phenomenologically by the dependence of the second term in the free-energy expression, Eq. (12), on the value of the net magnetic moment $J$. To discriminate between the impacts of the additive terms in 
Eq. (9) on the magnetization dynamics depicted in Fig. 8(b), we have plotted individual constituent parts. The normalized dynamics of the net magnetic moment is plotted in Fig. 8(d) and the normalized variation in concentration of the localized moments in Fig. 8(e). Similarly to the DMFT results, decay of the net magnetic moment $j$ demonstrates critical slowing-down in the vicinity of the Néel temperature. Slowing-down of the demagnetization time in the vicinity of the magnetic phase transition was observed in experiments on the iron-group transition-metal ferrimagnetic and ferromagnetic oxides [45,49-51], where the density of localized moments is fixed and $\Delta M$ is indeed proportional to $\Delta J$. We note that both of these features are not seen in our experiment. This again highlights that description of the observed magnetization dynamics only in terms of the net magnetic moment $J$ is insufficient. One can see that the light-induced variation in the concentration of the localized moments, presented in Fig. 8(e), is temperature independent and describes well the dynamics of the magnetization on a subpicosecond timescale. This underlines the role of delocalization effects in the ultrafast demagnetization observed in Mott insulators. Moreover, one can see that the fraction of the photodestructed moments is on the order of $1 \%$, which is significantly larger than the number of incident photons. This points out that in our experiment the delocalization is primarily dominated by the multiple carrier generation via the process of impact ionization.

\section{CONCLUSIONS}

Here, using the tabletop magneto-optical Kerr effect, we studied with high temporal resolution ultrafast spin and charge dynamics of the novel spin-orbit Mott insulator $\mathrm{Sr}_{2} \mathrm{IrO}_{4}$ having a net magnetic moment. We have shown that photodoping of $\mathrm{Sr}_{2} \mathrm{IrO}_{4}$ thin films by femtosecond laser pulses with photon energy well above the Mott gap drives an ultrafast response of both electronic and magnetic states. The magnetization dynamics probed by the MOKE appear to be substantially different from the charge dynamics probed by the photoinduced reflectivity. The Kerr effect reveals a melting of the antiferromagnetic order seen as ultrafast loss of the net magnetization occurring on a characteristic time of about $300 \mathrm{fs}$ and followed by a sub-10-ps recovery. At low temperature, the recovery of the reflectivity and the magneto-optical Kerr signal occur simultaneously. A temperature increase hardly affects the reflectivity dynamics, but results in a significant slowingdown of the dynamics of the magneto-optical signal. Supported by DMFT simulations and a phenomenological theory based on Onsager's formalism, we suggest that such long time of the ultrafast melting is primarily attributed to the gradual emergence of an excess amount of the holons and doublons in the course of the impact ionization. The picosecond magnetization recovery dynamics is a result of the relaxation of the magnetic system into the state corresponding to thermodynamic equilibrium and thus predominantly defined by the dependence of the magnetic potential on magnetization. All together, our work suggests a high potential of the tabletop magneto-optical setups for studying magnetic interactions in correlated electronic systems. Exploring the dependence of the magnetic correlations in the Mott systems to the wavelength of the pump excitation, resonant lattice, magnetic perturbation, etc., has not only considerable interest for experimental research but also a potential stimulus for further development of multiband DMFT and effective phenomenological models.

\section{ACKNOWLEDGMENTS}

The authors thank T. Toonen and S. Semin for technical support, and Y. M. Blanter and J. R. Hortensius for fruitful discussions. This work was partially supported by The Netherlands Organization for Scientific Research (NWO/OCW) as part of the Frontiers of Nanoscience program (NanoFront), and VENI-VIDI-VICI program, the European Union's Seventh Framework Program (FP7/ 2007-2013)/ERC Grant Agreement No. 339813 (Exchange), the program Leading Scientist of the Russian Ministry of Science and Higher Education (14.Z50.31.0034), by the EU through the European Research Council, Grant No. 677458 (AlterMateria), by the Ministry of Science and Higher Education of the Russian Federation in the framework of Increase Competitiveness Program of NUST MISiS (No. K2-2017-005), implemented by a governmental decree dated 16th of March 2013, N211, and by the National Academy of Sciences of Ukraine via Project No. 1/17-N.

[1] R. D. Averitt and A. J. Taylor, Ultrafast Optical and FarInfrared Quasiparticle Dynamics in Correlated Electron Materials, J. Phys. Condens. Matter 14, R1357 (2002).

[2] A. Kirilyuk, A. V. Kimel, and T. Rasing, Ultrafast Optical Manipulation of Magnetic Order, Rev. Mod. Phys. 82, 2731 (2010).

[3] J. Walowski and M. Münzenberg, Perspective: Ultrafast Magnetism and $\mathrm{THz}$ Spintronics, J. Appl. Phys. 120, 140901 (2016).

[4] B. A. Ivanov, Spin Dynamics of Antiferromagnets Under Action of Femtosecond Laser Pulses, Low Temp. Phys. 40, 91 (2014).

[5] E. Beaurepaire, J.-C. Merle, A. Daunois, and J.-Y. Bigot, Ultrafast Spin Dynamics in Ferromagnetic Nickel, Phys. Rev. Lett. 76, 4250 (1996).

[6] A. V. Kimel, R. V. Pisarev, J. Hohlfeld, and T. Rasing, Ultrafast Quenching of the Antiferromagnetic Order in $\mathrm{FeBO}_{3}$ : Direct Optical Probing of the Phonon-Magnon Coupling, Phys. Rev. Lett. 89, 287401 (2002).

[7] D. Khomskii, Transition Metal Compounds (Cambridge University Press, Cambridge, England, 2014).

[8] M. Imada, A. Fujimori, and Y. Tokura, Metal-Insulator Transitions, Rev. Mod. Phys. 70, 1039 (1998). 
[9] P. Werner, N. Tsuji, and M. Eckstein, Nonthermal Symmetry-Broken States in the Strongly Interacting Hubbard Model, Phys. Rev. B 86, 205101 (2012).

[10] J. H. Mentink and M. Eckstein, Ultrafast Quenching of the Exchange Interaction in a Mott Insulator, Phys. Rev. Lett. 113, 057201 (2014).

[11] K. Balzer, F. A. Wolf, I. P. McCulloch, P. Werner, and M. Eckstein, Nonthermal Melting of Néel Order in the Hubbard Model, Phys. Rev. X 5, 031039 (2015).

[12] M. Eckstein and P. Werner, Ultra-fast Photo-Carrier Relaxation in Mott Insulators with Short-Range Spin Correlations, Sci. Rep. 6, 21235 (2016).

[13] S. Dal Conte, L. Vidmar, D. Golež, M. Mierzejewski, G. Soavi, S. Peli, F. Banfi, G. Ferrini, R. Comin, B. M. Ludbrook et al., Snapshots of the Retarded Interaction of Charge Carriers with Ultrafast Fluctuations in Cuprates, Nat. Phys. 11, 421 (2015).

[14] M. Deb, P. Molho, B. Barbara, and J.-Y. Bigot, Controlling Laser-Induced Magnetization Reversal Dynamics in a RareEarth Iron Garnet Across the Magnetization Compensation Point, Phys. Rev. B 97, 134419 (2018).

[15] M. Dean, Y. Cao, X. Liu, S. Wall, D. Zhu, R. Mankowsky, V. Thampy, X. M. Chen, J. G. Vale, D. Casa et al., Ultrafast Energy- and Momentum-Resolved Dynamics of Magnetic Correlations in the Photo-Doped Mott Insulator $\mathrm{Sr}_{2} \mathrm{IrO}_{4}$, Nat. Mater. 15, 601 (2016).

[16] O. Krupin, G. L. Dakovski, B. J. Kim, J. W. Kim, J. Kim, S. Mishra, Y.-D. Chuang, C. R. Serrao, W. S. Lee, W. F. Schlotter et al., Ultrafast Dynamics of Localized Magnetic Moments in the Unconventional Mott Insulator $\mathrm{Sr}_{2} \mathrm{IrO}_{4}$, J. Phys. Condens. Matter 28, 32LT01 (2016).

[17] A. K. Zvezdin and V. A. Kotov, Modern Magnetooptics and Magnetooptical Materials (CRC Press, Boca Raton, USA, 1997).

[18] M. K. Crawford, M. A. Subramanian, R. L. Harlow, J. A. Fernandez-Baca, Z. R. Wang, and D. C. Johnston, Structural and Magnetic Studies of $\mathrm{Sr}_{2} \mathrm{IrO}_{4}$, Phys. Rev. B 49, 9198 (1994).

[19] Y. Gim, A. Sethi, Q. Zhao, J. F. Mitchell, G. Cao, and S. L. Cooper, Isotropic and Anisotropic Regimes of the FieldDependent Spin Dynamics in $\mathrm{Sr}_{2} \mathrm{IrO}_{4}$ : Raman Scattering Studies, Phys. Rev. B 93, 024405 (2016).

[20] H. Gretarsson, N. H. Sung, M. Höppner, B. J. Kim, B. Keimer, and M. Le Tacon, Two-Magnon Raman Scattering and Pseudospin-Lattice Interactions in $\mathrm{Sr}_{2} \mathrm{IrO}_{4}$ and $\mathrm{Sr}_{3} \mathrm{Ir}_{2} \mathrm{O}_{7}$, Phys. Rev. Lett. 116, 136401 (2016).

[21] B. J. Kim, H. Jin, S. J. Moon, J.-Y. Kim, B.-G. Park, C. S. Leem, J. Yu, T. W. Noh, C. Kim, S.-J. Oh et al., Novel $J_{\text {eff }}=1 / 2$ Mott State Induced by Relativistic Spin-Orbit Coupling in $\mathrm{Sr}_{2} \mathrm{IrO}_{4}$, Phys. Rev. Lett. 101, 076402 (2008).

[22] J. Kim, D. Casa, M. H. Upton, T. Gog, Y.-J. Kim, J. F. Mitchell, M. van Veenendaal, M. Daghofer, J. van den Brink, G. Khaliullin, and B. J. Kim, Magnetic Excitation Spectra of $\mathrm{Sr}_{2} \mathrm{IrO}_{4}$ Probed by Resonant Inelastic X-Ray Scattering: Establishing Links to Cuprate Superconductors, Phys. Rev. Lett. 108, 177003 (2012).

[23] H. Jin, H. Jeong, T. Ozaki, and J. Yu, Anisotropic Exchange Interactions of Spin-Orbit-Integrated States in $\mathrm{Sr}_{2} \mathrm{IrO}_{4}$, Phys. Rev. B 80, 075112 (2009).
[24] R. Peierls, On the Theory of the Diamagnetism of Conduction Electrons, in Selected Scientific Papers of Sir Rudolf Peierls, with Commentary by the Author, edited by H. Dalitz and R. Peierls (World Scientific, Singapore, 1997) pp. 97-120.

[25] F. Ye, S. Chi, B. C. Chakoumakos, J. A. Fernandez-Baca, T. Qi, and G. Cao, Magnetic and Crystal Structures of $\mathrm{Sr}_{2} \mathrm{IrO}_{4}$ : A Neutron Diffraction Study, Phys. Rev. B 87, 140406(R) (2013).

[26] L. Miao, H. Xu, and Z. Q. Mao, Epitaxial Strain Effect on the $J_{\text {eff }}=1 / 2$ Moment Orientation in $\mathrm{Sr}_{2} \mathrm{IrO}_{4}$ Thin Films, Phys. Rev. B 89, 035109 (2014).

[27] G. Cao, J. Bolivar, S. McCall, J. E. Crow, and R. P. Guertin, Weak Ferromagnetism, Metal-to-Nonmetal Transition, and Negative Differential Resistivity in Single-Crystal $\mathrm{Sr}_{2} \mathrm{IrO}_{4}$, Phys. Rev. B 57, R11039 (1998).

[28] A. Lupascu, J.P. Clancy, H. Gretarsson, Z. Nie, J. Nichols, J. Terzic, G. Cao, S.S. A. Seo, Z. Islam, M. H. Upton, Jungho Kim, D. Casa, T. Gog, A. H. Said, V. M. Katukuri, H. Stoll, L. Hozoi, J. van den Brink, and Y.-J. Kim, Tuning Magnetic Coupling in $\mathrm{Sr}_{2} \mathrm{IrO}_{4}$ Thin Films with Epitaxial Strain, Phys. Rev. Lett. 112, 147201 (2014).

[29] B. J. Kim, H. Ohsumi, T. Komesu, S. Sakai, T. Morita, H. Takagi, and T.-H. Arima, Phase-Sensitive Observation of a Spin-Orbital Mott State in $\mathrm{Sr}_{2} \mathrm{IrO}_{4}$, Science 323, 1329 (2009).

[30] F. J. Kahn, P. S. Pershan, and J. P. Remeika, Ultraviolet Magneto-Optical Properties of Single-Crystal Orthoferrites, Garnets, and Other Ferric Oxide Compounds, Phys. Rev. 186, 891 (1969).

[31] W. Wettling, Magneto-Optics of Ferrites, J. Magn. Magn. Mater. 3, 147 (1976).

[32] J. Suits, Faraday and Kerr Effects in Magnetic Compounds, IEEE Trans. Magn. 8, 95 (1972).

[33] J. Nichols, O. B. Korneta, J. Terzic, L. E. De Long, G. Cao, J. W. Brill, and S. S. A. Seo, Anisotropic Electronic Properties of a-Axis-Oriented $\mathrm{Sr}_{2} \mathrm{IrO}_{4}$ Epitaxial Thin-Films, Appl. Phys. Lett. 103, 131910 (2013).

[34] J. Nichols, J. Terzic, E. G. Bittle, O. B. Korneta, L. E. De Long, J. W. Brill, G. Cao, and S. S. A. Seo, Tuning Electronic Structure via Epitaxial Strain in $\mathrm{Sr}_{2} \mathrm{IrO}_{4}$ Thin Films, Appl. Phys. Lett. 102, 141908 (2013).

[35] S. A. Akhmanov, V. A. Vysloukh, and A. S. Chirkin, Optics of Femtosecond Laser Pulses (American Institute of Physics, New York, 1992).

[36] S. J. Moon, H. Jin, K. W. Kim, W. S. Choi, Y. S. Lee, J. Yu, G. Cao, A. Sumi, H. Funakubo, C. Bernhard et al., Dimensionality-Controlled Insulator-Metal Transition and Correlated Metallic State in 5d Transition Metal Oxides $\mathrm{Sr}_{n+1} \operatorname{Ir}_{n} \mathrm{O}_{3 n+1}(n=1,2$, and $\infty)$, Phys. Rev. Lett. 101, 226402 (2008).

[37] S. J. Moon, H. Jin, W. S. Choi, J. S. Lee, S. S. A. Seo, J. Yu, G. Cao, T. W. Noh, and Y. S. Lee, Temperature Dependence of the Electronic Structure of the $J_{\mathrm{eff}}=\frac{1}{2}$ Mott Insulator $\mathrm{Sr}_{2} \mathrm{IrO}_{4}$ Studied by Optical Spectroscopy, Phys. Rev. B 80, 195110 (2009).

[38] S. Iwai, M. Ono, A. Maeda, H. Matsuzaki, H. Kishida, H. Okamoto, and Y. Tokura, Ultrafast Optical Switching to a Metallic State by Photoinduced Mott Transition in a 
Halogen-Bridged Nickel-Chain Compound, Phys. Rev. Lett. 91, 057401 (2003).

[39] D. Wegkamp, M. Herzog, L. Xian, M. Gatti, P. Cudazzo, C. L. McGahan, R. E. Marvel, R. F. Haglund, A. Rubio, M. Wolf, and J. Stähler, Instantaneous Band Gap Collapse in Photoexcited Monoclinic $\mathrm{VO}_{2}$ due to Photocarrier Doping, Phys. Rev. Lett. 113, 216401 (2014).

[40] G. Yu, C. H. Lee, A. J. Heeger, N. Herron, and E. M. McCarron, Transient Photoinduced Conductivity in Single Crystals of $\mathrm{YBa}_{2} \mathrm{Cu}_{3} \mathrm{O}_{6.3}$ : Photodoping to the Metallic State, Phys. Rev. Lett. 67, 2581 (1991).

[41] H. Okamoto, T. Miyagoe, K. Kobayashi, H. Uemura, H. Nishioka, H. Matsuzaki, A. Sawa, and Y. Tokura, Photoinduced Transition from Mott Insulator to Metal in the Undoped Cuprates $\mathrm{Nd}_{2} \mathrm{CuO}_{4}$ and $\mathrm{La}_{2} \mathrm{CuO}_{4}$, Phys. Rev. $\mathrm{B}$ 83, 125102 (2011).

[42] H. Yamakawa, T. Miyamoto, T. Morimoto, T. Terashige, H. Yada, N. Kida, M. Suda, H. M. Yamamoto, R. Kato, K. Miyagawa et al., Mott Transition by an Impulsive Dielectric Breakdown, Nat. Mater. 16, 1100 (2017).

[43] P. Werner, K. Held, and M. Eckstein, Role of Impact Ionization in the Thermalization of Photoexcited Mott Insulators, Phys. Rev. B 90, 235102 (2014).

[44] S. Mathias, C. La-O-Vorakiat, P. Grychtol, P. Granitzka, E. Turgut, J. M. Shaw, R. Adam, H. T. Nembach, M. E. Siemens, S. Eich, C. M. Schneider, T. J. Silva, M. Aeschlimann, M. M. Murnane, and H. C. Kapteyn, Probing the Timescale of the Exchange Interaction in a Ferromagnetic Alloy, Proc. Natl. Acad. Sci. U.S.A. 109, 4792 (2012).
[45] T. Ogasawara, K. Ohgushi, Y. Tomioka, K. S. Takahashi, H. Okamoto, M. Kawasaki, and Y. Tokura, General Features of Photoinduced Spin Dynamics in Ferromagnetic and Ferrimagnetic Compounds, Phys. Rev. Lett. 94, 087202 (2005).

[46] L. D. Landau and E. M. Lifshitz, Statistical Physics: V. 5: Course of Theoretical Physics (Pergamon Press, New York, 1969).

[47] J. H. Mentink, J. Hellsvik, D. V. Afanasiev, B. A. Ivanov, A. Kirilyuk, A. V. Kimel, O. Eriksson, M. I. Katsnelson, and T. Rasing, Ultrafast Spin Dynamics in Multisublattice Magnets, Phys. Rev. Lett. 108, 057202 (2012).

[48] L. D. Landau and I. M. Khalatnikov, On the Anomalous Absorption of Sound Near a Second Order Phase Transition, Dokl. Akad. Nauk SSSR 96, 469 (1954).

[49] C. L. S. Kantner, M. C. Langner, W. Siemons, J. L. Blok, G. Koster, A. J. H. M. Rijnders, R. Ramesh, and J. Orenstein, Determination of the Spin-Flip Time in Ferromagnetic $\mathrm{SrRuO}_{3}$ from Time-Resolved Kerr Measurements, Phys. Rev. B 83, 134432 (2011).

[50] T. Kise, T. Ogasawara, M. Ashida, Y. Tomioka, Y. Tokura, and M. Kuwata-Gonokami, Ultrafast Spin Dynamics and Critical Behavior in Half-Metallic Ferromagnet: $\mathrm{Sr}_{2} \mathrm{FeMoO}_{6}$, Phys. Rev. Lett 85, 1986 (2000).

[51] J. P. Hinton, S. Patankar, E. Thewalt, A. Ruiz, G. Lopez, N. Breznay, A. Vishwanath, J. Analytis, J. Orenstein, J. D. Koralek et al., Photoexcited States of the Harmonic Honeycomb Iridate $\gamma-\mathrm{Li}_{2} \mathrm{IrO}_{3}$, Phys. Rev. B 92, 115154 (2015). 